\title{
Immigrants and borderline personality disorder at a psychiatric emergency service
}

\author{
J. C. Pascual, A. Malagón, D. Córcoles, J. M. Ginés, J. Soler, C. García-Ribera, V. Pérez
}

and A. Bulbena

\section{Background}

Several studies have suggested that immigrants have higher rates of psychiatric emergency service use and a higher risk of mental disorders such as schizophrenia than indigenous populations.

\section{Aims}

To compare the likelihood that immigrants (immigrant group) $v$. indigenous population (indigenous group) will be diagnosed with borderline personality disorder in a psychiatric emergency service and to determine differences according to area of origin.

\section{Method}

A total of 11578 consecutive admissions over a 4-year period at a tertiary psychiatric emergency service were reviewed. The collected data included socio-demographic and clinical variables and the Severity of Psychiatric Illness rating score. Psychiatric diagnosis was limited to information available in the emergency room given that a structured interview is not usually feasible in this setting. The diagnosis of borderline personality disorder was based on DSM-IV criteria. Immigrants were divided into five groups according to region of origin: North Africa, sub-Saharan Africa, South America, Asia and Western countries.

\section{Results}

Multivariate statistical logistic regression analysis showed that all subgroups of immigrants had a lower likelihood of being diagnosed with borderline personality disorder than the indigenous population independently of age and gender. Furthermore, the rates of borderline personality disorder diagnosis were considerably lower in Asian and sub-Saharan subgroups than in South American, North African, Western or native subgroups.

\section{Conclusions}

Our results showed that in the psychiatric emergency service borderline personality disorder was diagnosed less frequently in the immigrant group $v$. the indigenous group. Our results do not support the concept of migration as a risk factor for borderline personality disorder.

\section{Declaration of interest}

None. Funding detailed in Acknowledgements.
Spain has recently changed from being a country of emigration to one of immigration and in the past 5 years the number of immigrants has increased dramatically. The percentage of immigrants in Barcelona is currently $14 \%$ of the total population and in some areas of the city this rate reaches $38 \% .^{1}$

Several studies have suggested that immigration could be a risk factor for mental health disorders such as psychosis and that immigrants present to psychiatric emergency services at higher rates than indigenous patients. ${ }^{2-4}$ However, other reports do not support these findings and argue that immigrants present a lower risk of psychiatric disorders such as alcohol and drug use disorders, major depression, dysthymia, mania, or anxiety disorders, ${ }^{5-7}$ and tend to underuse psychiatric services. ${ }^{89}$ In some of these studies immigrant samples have been analysed as a single group and major cultural differences related to region of origin are not taken into account.

The association between personality disorder and migration or ethnicity has not been extensively investigated. Although several authors have found lower rates of personality disorder among Black and minority ethnic patients compared with White patients in forensic and non-forensic psychiatric services, ${ }^{10,11}$ we found only two studies that analysed the relationship between immigration and personality disorders in psychiatric emergency services. Tyrer et $a l^{12}$ found a lower incidence of personality disorder among African-Caribbean patients compared with White patients presenting to a psychiatric emergency service in London, and Baleydier et $a l^{13}$ reported a lower frequency of a general personality disorder in immigrants presenting at a psychiatric emergency service in Geneva when compared with indigenous patients. Paris ${ }^{14}$ suggests that immigration itself could be a social risk factor for borderline personality disorder and that some immigrants from traditional societies without borderline pathology in their country of origin might develop the disorder in a modern society such as North America. The aims of this study were to examine the association between immigration and borderline personality disorder in a Spanish psychiatric emergency service and to investigate possible differences in relation to the region of origin.

\section{Method}

We evaluated a total of 11578 consecutive admissions seen over a 4-year period at the psychiatric emergency service of a tertiary university hospital in the city of Barcelona, Spain. This hospital is part of the national health service and provides free medical service $24 \mathrm{~h}$ per day to the indigenous population and immigrants, regardless of legal status. The hospital's catchment area serves a population of 332000 and has the highest immigrant concentration in Barcelona.

Written consent to participate in the study was not obtained as information was collected from routine admission data, but the principles outlined in the Declaration of Helsinki were followed. The study was approved by the clinical research ethics committee at the Hospital del Mar.

\section{Participants}

We studied all admissions following a routine computerised protocol that comprised demographic and clinical variables. ${ }^{15,16}$ Socio-demographic data included age, gender and country of 
origin. Clinical characteristics included: reason for seeking care (grouped into six categories: psychosis, anxiety, depression, disruptive behaviour, drug misuse or other), referral source (self-referrals, ambulance, police or other), previous psychiatric history, previous contact with mental health services, substance misuse or dependence, diagnosis and hospitalisation. It is not usually possible to administer semi-structured diagnostic instruments in a psychiatric emergency service setting. Diagnosis was therefore made based on information gathered from the patient and family, and from any computerised out-patient psychiatric data available at the centre. Because patients can have more than one psychiatric diagnosis, we grouped the major mental illnesses into six categories: psychotic, affective, anxiety, drug misuse, personality (including borderline personality disorder), and other Axis I disorders. For the purpose of this study, borderline personality disorder diagnosis was made at the time of admission using the information collected, systematically assessing each DSM-IV criteria ${ }^{17}$ for the disorder. Comorbid diagnoses were not analysed as each admission received only one main diagnosis. 'Immigrants' were defined as any foreign-born individuals, regardless of whether or not they had been granted Spanish nationality. Tourists were not included in the sample. We divided the immigrants into five groups according to region of origin: North Africa, sub-Saharan Africa, South America, Asia, and Western countries (Europe, USA, Canada and Australia).

Severity of illness was assessed by the Spanish version of the Severity of Psychiatric Illness (SPI) scale. ${ }^{18,19}$ This instrument was developed as a patient-level decision support tool to assess the need for services - especially in-patient care - based on each patient's clinical and social factors. It is a clinician-administered rating scale consisting of 12 items that are scored on a four-point scale from 0 (absent) to 3 (severe problem). Specific domains include suicide risk, danger to others, severity of symptoms, difficulty with self-care, medical problems, drug problems, job problems, family disruption, housing instability, treatment adherence, family involvement, and premorbid dysfunction. The SPI was completed by the clinician at the end of the psychiatric emergency service visit based on all available information (from patients, family and clinical records). All psychiatric emergency staff had attended an SPI training programme.

\section{Statistical analysis}

Data were analysed using the SPSS 14.0 software package for Windows. Over the 4-year period, a single patient may have been seen more than once at the psychiatric emergency service. However, as their socio-demographic and clinical characteristics (e.g. diagnosis, reason for referral, SPI scores) possibly varied between consultations, the unit of analyses used was each separate episode of care rather than the individual patient. All hypotheses were tested at a two-sided, 0.05 significance level. Demographic and clinical characteristics of immigrants (immigrant group) and indigenous patients (indigenous group) were compared using chi-squared tests for categorical variables and Student's $t$-tests for continuous variables. Items on the SPI scale were compared between the two groups using the non-parametric Mann-Whitney $U$-test. We also analysed differences between each subgroup of immigrants in comparison with the indigenous group.

The relationship between immigrant subgroups and borderline personality disorder diagnosis was examined by multivariate logistic regression analysis. The dependent variable was borderline personality disorder diagnosis and the independent variables were all subgroups of immigrant status. Age and gender were included as potential confounding variables.

\section{Results}

Of the 11578 admissions to the psychiatric emergency service, $1345(11.6 \%)$ were immigrants: 35\% were from South America, 29\% from North African, 24\% from Western countries, $8 \%$ from Asia and 4\% from sub-Saharan Africa. A total of 250 admissions were excluded from the study because they were tourists.

\section{Differences between immigrant and indigenous patients}

Online Table DS1 summarises demographic and clinical characteristics and shows the differences between the immigrant and indigenous groups. Compared with the indigenous group, immigrants were younger and more frequently males. Forty-three per cent of immigrant patients presented serious social problems and $11 \%$ had a language barrier. They had fewer psychiatric antecedents and less previous contact with out-patient mental health services. There were no differences between immigrant and indigenous groups with respect to substance use disorder. Hospitalisation was higher for immigrants than for the indigenous group. Immigrants were more frequently brought to the psychiatric emergency service by ambulance or police. Rates of borderline personality disorder diagnosis among immigrants were lower than in the indigenous sample: $5.7 \%$ v. 9.5\% respectively. The SPI scale showed that compared with the indigenous group, immigrants had lower levels of symptom severity, suicide risk and medical problems and a similar risk of danger to others and drug problems. However, they showed more difficulties related to job, family and housing.

\section{Differences between subgroups of immigrant patients}

Table 1 shows demographic and clinical variables for each subgroup of immigrants compared with the indigenous group. Table 2 presents differences in SPI scores. All subgroups of immigrants showed lower rates of borderline personality disorder diagnosis than the indigenous group. However, rates in the subgroups of patients from South America, North Africa and Western countries were more similar to those of the indigenous sample than those in the Asian and sub-Saharan African subgroups.

Patients from North Africa manifested more substance misuse and were more frequently brought to the psychiatric emergency service by police. Hospitalisation was also higher in this subgroup. The reason for emergency consultation was more frequently disruptive behaviour, and depression was infrequent. These patients had a higher risk of danger to others but lower severity of symptoms and fewer medical problems. They had more difficulties related to job, family and housing than the indigenous group.

The sub-Saharan African subgroup had a similar rate of drug problems when compared with the indigenous group. The main reason for consultation was psychotic symptoms and there were more hospitalisations in this subgroup than in the indigenous group. They did not present risk of suicide but had a greater risk of danger to others and more job, family and housing difficulties.

The South American subgroup was the only immigrant group with a greater proportion of females than males. They had fewer drug problems but more anxiety symptoms than the indigenous group. Total SPI scores were lower and they showed less severity of symptoms, less risk of suicide, lower risk of danger to others and more self-care ability. However, they had more job and family problems than the indigenous group.

The Asian subgroup presented with fewer drug problems and were more frequently brought to the psychiatric emergency service 
Table 1 Comparisons of demographic and clinical characteristics between subgroups of immigrants and the indigenous group

\begin{tabular}{|c|c|c|c|c|c|c|}
\hline \multirow[b]{2}{*}{ Variable } & \multirow[b]{2}{*}{$\begin{array}{l}\text { Indigenous group } \\
\qquad(n=9983)\end{array}$} & \multicolumn{5}{|c|}{ Immigrant subgroup } \\
\hline & & $\begin{array}{l}\text { North Africa } \\
(n=395)\end{array}$ & $\begin{array}{c}\text { Sub-Saharan Africa } \\
\qquad(n=51)\end{array}$ & $\begin{array}{l}\text { South America } \\
\qquad(n=475)\end{array}$ & $\begin{array}{c}\text { Asia } \\
(n=105)\end{array}$ & $\begin{array}{l}\text { Western countries } \\
\qquad(n=319)\end{array}$ \\
\hline Age, years: ${ }^{a}$ mean (s.d.) & $41.38(15.6)$ & $31.33(10.3)^{\star * *}$ & $29.75(8)^{* * *}$ & $35.06(12.1)^{\star \star *}$ & $34.77(11.2)^{* * *}$ & $32.45(10.5)^{* * *}$ \\
\hline Male, ${ }^{\mathrm{b}} \%$ & 46.4 & $71.6^{* * *}$ & $68.6^{* *}$ & $34.9^{* * *}$ & $66.7^{* * *}$ & $52.0^{*}$ \\
\hline Previous psychiatry history, $\%$ & 81.5 & $61.7 * * *$ & $52.9^{* * *}$ & $59.2^{\star * \star}$ & $54.3^{* * *}$ & $69.6^{* * *}$ \\
\hline Previous psychiatry service contact, ${ }^{\text {, }} \%$ & 75.4 & $53.1 * * *$ & $51.0 * * *$ & $46.1^{* * *}$ & $41 * * *$ & $54.5^{\star * *}$ \\
\hline Drug misuse, ${ }^{\mathrm{b}} \%$ & 30 & $43^{* * *}$ & 30 & $21.1^{* *}$ & 25.3 & 32.8 \\
\hline \multicolumn{7}{|l|}{ Referral source, ${ }^{\text {b } \%}$} \\
\hline Self & 55.9 & $41.6^{* * *}$ & $31.4^{* \star *}$ & 58.3 & $41.9^{* *}$ & $49.2^{*}$ \\
\hline Ambulance & 34.2 & $39.3^{* *}$ & $56.9 * * *$ & 32.2 & $46.7 * * *$ & 36.4 \\
\hline Police & 2.0 & $10.7^{* * *}$ & 5.9 & 2.7 & 1.0 & $6.6^{* * *}$ \\
\hline Others & 7.9 & 8.4 & 5.9 & 6.7 & 10.5 & 7.5 \\
\hline \multicolumn{7}{|l|}{ Reason for referral, \% } \\
\hline Depression & 20.1 & $9.9 * * *$ & $5.9 * *$ & 22.5 & $10.5^{*}$ & 16.3 \\
\hline Anxiety & 27.1 & 29.1 & 17.6 & $32.8^{\star *}$ & 31.4 & 28.5 \\
\hline Psychosis & 8.8 & $12.4^{*}$ & $29.4^{* * *}$ & 8.4 & $15.2^{*}$ & 10.7 \\
\hline Disruptive behaviour & 14.2 & $22.8 * * *$ & 21.6 & 14.9 & $21.9^{*}$ & 14.1 \\
\hline Drug misuse & 8.7 & 9.1 & 9.8 & $4.2^{\star \star \star *}$ & 4.8 & 10.0 \\
\hline Others & 21.1 & $16.7^{*}$ & 15.7 & 17.1 & 16.2 & 20.4 \\
\hline Hospitalisation, ${ }^{\mathrm{b}} \%$ & 15.8 & $21.9^{* * *}$ & $49.0^{* * *}$ & 14.5 & $29.5^{* * *}$ & 18.2 \\
\hline $\begin{array}{l}\text { Borderline personality disorder } \\
\text { diagnosis, } n(\%)\end{array}$ & $948(9.5 \%)$ & $25(6.3 \%)^{*}$ & $1(2 \%)^{* * *}$ & $27(5.7)^{\star *}$ & $3(2.9)^{* * *}$ & $20(6.3 \%)^{*}$ \\
\hline $\begin{array}{l}\text { a. } t \text {-test. } \\
\text { b. Chi-squared test. } \\
\star P<0.05 ; * * P<0.01 ; * * * P<0.001 \text {. }\end{array}$ & & & & & & \\
\hline
\end{tabular}

\begin{tabular}{|c|c|c|c|c|c|c|}
\hline \multirow[b]{2}{*}{ Severity of Psychiatric Illness itema } & \multirow[b]{2}{*}{$\begin{array}{l}\text { Indigenous group } \\
\qquad(n=9983)\end{array}$} & \multicolumn{5}{|c|}{ Immigrant subgroup } \\
\hline & & $\begin{array}{l}\text { North Africa } \\
\qquad(n=395)\end{array}$ & $\begin{array}{l}\text { Sub-Saharan Africa } \\
\qquad(n=51)\end{array}$ & $\begin{array}{l}\text { South America } \\
\qquad(n=475)\end{array}$ & $\begin{array}{c}\text { Asia } \\
(n=105)\end{array}$ & $\begin{array}{l}\text { Western countries } \\
\qquad(n=319)\end{array}$ \\
\hline Suicide risk ( $\geqslant 2$ ), \% & 7.0 & 4.8 & $0 *$ & $3.8^{* \star}$ & 8.6 & 5.0 \\
\hline Danger to others $(\geqslant 1), \%$ & 28.0 & $38.0^{\star \star \star *}$ & $52.0^{\star * \star}$ & $23.3^{*}$ & 34.3 & 24.5 \\
\hline Severity of symptoms $(\geqslant 2), \%$ & 56.2 & $48.0^{* \star}$ & 62.0 & $50.5^{\star}$ & 53.3 & $49.2^{*}$ \\
\hline Self-care ability $(\geqslant 1), \%$ & 57.9 & $51.3^{* *}$ & 68.0 & $41.3^{* * \star}$ & 50.5 & $49.1^{* *}$ \\
\hline Medical problems $(\geqslant 2), \%$ & 18.9 & $8.7 * * *$ & 14.0 & $8.1^{* * *}$ & 12.4 & $10.4^{* * *}$ \\
\hline Drug problems ( $\geqslant 2$ ), $\%$ & 25.7 & $36.0^{* \star \star}$ & 36.0 & $16.7^{* \star \star}$ & 21.9 & 26.7 \\
\hline Job problems $(\geqslant 2), \%$ & 20.2 & $45.1 * * *$ & $48.0^{* \star *}$ & $31.6^{* * *}$ & 26.7 & $37.1 * * *$ \\
\hline Family disruption $(\geqslant 2), \%$ & 38.6 & $53.8^{\star \star \star *}$ & 50.0 & $47.9 * \star \star$ & 32.4 & 42.0 \\
\hline Home instability $(\geqslant 2), \%$ & 11.0 & $29.1 * * *$ & $38.0^{* * \star}$ & 11.7 & 16.2 & $28.0^{* \star *}$ \\
\hline Treatment adherence $(\geqslant 3), \%$ & 4.2 & 4.6 & 2.0 & 2.5 & 5.7 & 5.7 \\
\hline Family involvement ( $\geqslant 2), \%$ & 18.4 & $35.5^{* * *}$ & $44.0^{* \star *}$ & $22.5^{*}$ & 21.9 & $30.8^{* * *}$ \\
\hline Premorbid dysfunction $(\geqslant 2), \%$ & 33.4 & 29.8 & 24.0 & $18.5^{* \star \star}$ & 25.7 & $21.1^{* \star \star}$ \\
\hline Total, mean (s.d.) & $9.14(5.1)$ & $10.88(6)^{\star * \star}$ & $11.82(4.9)^{\star \star \star}$ & $8.4(4.7)^{\star \star}$ & $9.15(6)$ & $9.92(5.5)^{\star *}$ \\
\hline
\end{tabular}

by ambulance than the indigenous group. The main reasons for consultation were disruptive behaviour and psychotic symptoms. Depression was less frequent. The proportion of hospitalisation was higher than for the indigenous group. Scores for all items of the SPI were similar to those for the indigenous group.

Finally, immigrants from Western countries presented clinical characteristics that were similar to those of the indigenous group but they had more job, family and housing difficulties.

\section{Association between immigration and borderline personality disorder}

Table 3 shows the final logistic regression model that included the immigrant subgroups, age and gender as independent variables.
The logistic regression was significant for all variables. Younger patients were more likely to be attributed a diagnosis of borderline personality disorder than older patients. Men had less risk of receiving a diagnosis of borderline personality disorder than women. All subgroups of immigrants were less likely to be diagnosed as having the disorder than the indigenous group, independently of age and gender: the sub-Saharan African subgroup had more than seven times less chance of being diagnosed with the disorder; the Asian subgroup showed more than four times less risk; and North Africans, South Americans and Westerners presented about two times less risk.

To explore the cross-cultural validity of borderline personality disorder diagnoses, we compared patients with the disorder according to region of origin. We analysed age, gender and the 


\begin{tabular}{|c|c|c|}
\hline Variable & OR $(95 \% \mathrm{Cl})$ & $P$ \\
\hline Age, years & $0.94(0.94-0.95)$ & $<0.001$ \\
\hline Male & $0.57(0.50-0.65)$ & $<0.001$ \\
\hline \multicolumn{3}{|l|}{ Immigrant status } \\
\hline North Africa & $0.46(0.30-0.70)$ & $<0.001$ \\
\hline Sub-Saharan Africa & $0.13(0.02-0.94)$ & 0.044 \\
\hline South America & $0.40(0.27-0.60)$ & $<0.001$ \\
\hline Asia & $0.24(0.08-0.74)$ & 0.015 \\
\hline Western countries & $0.43(0.27-0.68)$ & $<0.0001$ \\
\hline
\end{tabular}

SPI items that are usually related with 'borderline personality disorder symptoms' such as suicide risk, danger to others, drug problems and family disruption. We found that all patients were similar independent of region of origin. There was no significant difference between subgroups in gender, risk of suicide, risk of danger to others or family disruption. However, patients with borderline personality disorder from North Africa and sub-Saharan Africa were younger, and those from Asia were older than the indigenous group. Only patients with the disorder from Western countries had significantly fewer drug problems than indigenous patients with borderline personality disorder.

\section{Discussion}

We found significant clinical differences between immigrant and indigenous patients that attended a psychiatric emergency service. We also observed differences between subgroups of immigrants in relation to geographical origin. Although immigrants from South America and Western countries presented clinical characteristics that were similar to the indigenous population, immigrants from African and Asian countries differed considerably. We also found that borderline personality disorder was diagnosed by the emergency psychiatric clinicians less frequently in immigrants than in the indigenous population. Moreover, subgroups from Asia and sub-Saharan Africa were attributed this diagnosis less frequently than other subgroups of immigrants.

\section{Differences between immigrant and indigenous patients}

The finding that immigrant patients were younger and predominantly male has been reported previously ${ }^{4}$ and can be expected given that, in general, young men are more likely to migrate than women. Immigrants had less previous contact with the ambulatory psychiatric care system than the indigenous group and were more often brought by ambulance or non-voluntarily by the police. Previous studies have also shown that immigrants do not seek care through the usual psychiatric circuits and take longer to seek help. ${ }^{4,20}$ A delay in care seeking could explain why, in our setting, immigrants showed higher illness severity when seen via the psychiatric emergency service and were more often brought involuntarily. Differences in cultural conceptualisation of mental illnesses could also account for the fact that they themselves do not seek help when they have psychiatric symptoms. ${ }^{20}$

The higher percentage of hospitalisations among immigrants when compared with the indigenous group could be related to the fact that when immigrants arrived at the psychiatric emergency service, they generally presented more severe symptoms, with higher levels of psychotic symptomatology and a greater risk of danger to others. This could perhaps be related to the unstructured environment in which immigrants often find themselves.

A relationship between migration and psychiatric disorders has not been clearly established. Although a recent meta-analysis found that migration was an important risk factor for the development of schizophrenia, ${ }^{3}$ other studies have reported a lower risk of psychiatric disorders such as alcohol and drug misuse, major depression, dysthymia, mania and anxiety disorders in immigrant populations. ${ }^{5-7}$ In another recent meta-analysis, migration was not found to be a risk factor for mood disorders. ${ }^{21}$ In our study, immigrants presented more psychotic symptoms but fewer depressive symptoms than the indigenous patients. As in other studies, we also found that immigrants were unlikely to seek medical help for depression. ${ }^{20}$ It is possible that these patients do not seek medical care as they do not consider their symptoms as an illness. It is important to point out that in agreement with other studies, we found that immigrants had a lower risk of suicide. $^{4}$

\section{Differences between subgroups of immigrant patients}

Differences among immigrants according to geographical origin have been reported previously by other authors. ${ }^{4,21}$ The lack of difference between the South American subgroup and the indigenous group in our study could be related to the fact that cultural and linguistic similarities may reduce the stress of acculturation in this subgroup. Patients from Western countries also presented few problems regarding integration. In general, they do not find marked cultural differences and do not therefore present severe adaptation problems. Furthermore, the medical and psychiatric healthcare system in their country of origin is generally comparable to that in Spain, as are generally attitudes to and beliefs about mental illness.

In contrast, cultural differences in subgroups of patients from African and Asian countries are more marked. They generally present behavioural disorders and psychotic symptoms, but they do not usually consider depressive symptoms to be an illness and rarely seek treatment for such disorders. ${ }^{4,22}$ It is also possible that they may use alternative medicine practices, such as acupuncture or herbal medicine.

\section{Association between immigration and borderline personality diagnosis}

Our findings support two previous studies that analysed rates of personality disorder among minority ethnic and foreign patients in a psychiatric emergency service. Tyrer et al ${ }^{12}$ found that the incidence of personality disorder among African-Caribbean patients attending a psychiatric emergency service (25\%) was lower than among White patients (63\%), and Baleydier et al ${ }^{13}$ reported that foreign patients seen at a psychiatric emergency service in Geneva were less likely to be given this diagnosis than the indigenous population.

There may be several possible explanations for this lower risk of diagnosis in certain immigrant groups. The first is the possibility that this is a true finding. Although epidemiological data are as yet lacking in areas of Asia and Africa, authors such as Murphy ${ }^{23}$ and Paris ${ }^{14}$ suggest that traditional cultures may provide protective factors against symptoms such as impulsive or parasuicide acts. These societies are likely to provide protective rules, values and roles that tend to induce acceptance of family 
and community expectations and inhibit emotional expression. Western societies, on the other hand, offer more flexible values and rules, favouring individualism and greater emotional expression. ${ }^{14}$ According to Linehan, achievement of personal goals is considered a priority, whereas a lack of motivation or discipline is associated with failure, a factor that could play a role in borderline personality disorder development in the Western world. $^{24}$ Millon $^{25}$ and Paris $^{26}$ consider that the increased prevalence of the disorder in Western countries in recent decades may also be due to rapid social change and the breakdown of community norms. Based on this argument, Paris suggests that immigration could be a trigger factor of borderline personality disorder as some patients who did not present symptoms in their country of origin developed them on immigrating to modern societies. Our results, however, do not coincide with this hypothesis as we found a lower risk of being diagnosed with borderline personality disorder among immigrants. Nevertheless, it should perhaps be taken into consideration that as immigration is a relatively new phenomenon in Spain, the immigrants evaluated in our centre are not yet totally immersed in their newly adopted society. Future studies in younger immigrants and second generations who will be more influenced by the Western way of life may be interesting and help clarify this. Another point that cannot be overlooked is the possibility that a lower incidence of borderline personality disorder diagnosis in immigrants could be related to the fact that those individuals who migrate might have a more 'resilient' personality than their counterparts who do not migrate.

As a second explanation, it cannot be ruled out that our findings might be explained by some methodological artefact in the diagnosis. If diagnosis of borderline personality disorder is particularly difficult in a psychiatric emergency service, it is even more complicated when there are cross-cultural differences. Our findings could be influenced by several types of bias: cross-cultural construct bias (originating from differences across cultures in the constructs being measured), cross-cultural method bias (as the result of administering the instrument across cultures), or crosscultural item bias (from the items being used as indicators of these constructs). A bias in cross-cultural clinical judgement is yet another valid possibility. ${ }^{27,28}$ However, the cross-cultural validity of borderline personality disorder diagnosis in our study was found to be acceptable, as all patients presented similar characteristics regardless of their country of origin. The differences between subgroups of immigrants could have been partly the result of a language barrier and/or misperception of culturally appropriate expression. Groups with the lowest risk of diagnoses, for example, had a more severe language barrier. However, clinicians had two resources at their disposal to deal with these communication difficulties. First, the hospital uses a network of foreign interpreters to help in clinical interviews when required, and second, the local health authorities provide a 24 -h telephone interpreter system. Therefore, we consider it very unlikely that lower rates of borderline personality disorder diagnosis in patients of African and Asian origin are the result of diagnostic errors due to idiomatic difficulties. It should also be taken into consideration that different ethnic groups may express emotions and distress differently. In our sample, the sub-Saharan African subgroup did not express suicide risk or depressive symptoms, but it did show disruptive behaviour. In a study in the USA, Chavira et $a l^{29}$ found that patients from South America presented higher levels of borderline personality disorder diagnosis than White or African American patients. In our setting, however, the South American subgroup had a similar percentage of the disorder to the indigenous group, possibly owing to their cultural similarities with Spain.
A final explanation could be that some subgroups do not seek help for typical borderline personality disorder symptoms. At present, in Spain, certain subgroups of immigrants, particularly those from Asian and sub-Saharan African countries, are more likely to have illegal status, and they are therefore less likely to voluntarily seek mental healthcare than the indigenous population or legal immigrants. Patients in these subgroups were more frequently brought to the psychiatric emergency service involuntarily and they usually present severe symptoms such as psychotic disorders or disruptive behaviour. The rates of borderline personality disorder diagnosis were therefore proportionally lower. Since, in our study, only one main diagnosis was assigned to each patient, in the presence of a severe psychotic disorder borderline personality disorder may have been overshadowed.

\section{Study limitations}

This study has several limitations. First, the reliability of diagnosis using an unstructured interview has been found to be poor ${ }^{30}$ and is probably even lower in the case of the cross-cultural diagnosis of personality disorder. ${ }^{31}$ Psychiatric diagnosis was based only on the information available at the emergency room, as a complete structured interview is not usually feasible in this setting. Although this is a major limitation of the study, prior studies appear to indicate that diagnoses made by clinicians in the psychiatric emergency service setting are highly reliable. ${ }^{32}$ Second, as the study is based only on individuals who visited a psychiatric emergency service, it is very likely that the sample is not representative of the total immigrant population. Third, cross-cultural biases may play a role in the differences observed; however, we consider that the availability of interpreters at our centre helped to minimise this. Fourth, comorbid diagnoses were not analysed because each admission received only one main diagnosis. Some patients with diagnoses of affective, anxiety or drug use disorders possibly also had borderline personality disorder. And finally, as our study reflects patterns from a single urban emergency unit in Spain, its generalisation to other countries is likely to be limited.

\section{Conclusions}

Although migration is often considered a risk factor for mental health disorders such as schizophrenia, our current findings do not support the concept of migration as a risk factor for borderline personality disorder. Our results indicate that the emergency psychiatric clinicians diagnosed this disorder in immigrant patients less frequently than in the indigenous group, and that the Asian and sub-Saharan African subgroups presented lower rates of borderline personality disorder diagnosis than other subgroups. Further studies designed to analyse environmental risk factors such as immigrant status may contribute further to our understanding of this highly prevalent disorder.

\section{J. C. Pascual, PhMDD Department of Psychiatry, Sta. Creu i St. Pau Hospital, Universitat Autònoma de Barcelona, and CIBER-SAM; A. Malagón, MD, D. Córcoles, MD, J. M. Gines, MD, Department of Psychiatry, IAPS, Hospital del Mar, Universitat Autònoma de Barcelona; J. Soler, PsyD, Department of Psychiatry, Sta. Creu i St. Pau Hospital, Universitat Autònoma de Barcelona, and CIBER-SAM; C. García-Ribera, MD, Department of Psychiatry, IAPS, Hospital del Mar, Universitat Autònoma de Barcelona; v. Pérez, PhD, MD, Department of Psychiatry, Sta. Creu i St. Pau Hospital, Universitat Autònoma de Barcelona, and CIBER-SAM; A. Bulbena, PhD, MD, Department of Psychiatry, IAPS, Hospital del Mar, Universitat Autònoma de Barcelona, Spain}

Correspondence: Dr J.C. Pascual, Department of Psychiatry, Hospital de la Santa Creu i Sant Pau, Avenida Sant Antoni M. Claret, 167. 08025 Barcelona, Spain. Email: jpascual@hsp.santpau.es

First received 22 Mar 2007, final revision 16 Jan 2008, accepted Feb 2008 


\section{Acknowledgements}

We thank the psychiatrists at the emergency service at Hospital del Mar for their participation in the study. This study was supported by grants from the Fondo de Investigación Sanitaria (Ministry of Health, Spain).

\section{References}

1 Ajuntament de Barcelona. La Població Estrangera a Barcelona. Informes Estadístics [Foreign Population in Barcelona. Statistical Report]. Department d'Estadística, 2006.

2 Ödegaard Ö. Emigration and insanity. A study of mental disease among Norwegian-born population in Minnesota. Acta Psychiatr Neurol Scand Suppl 1932; 4: 1-206.

3 Cantor-Graae E, Selten JP. Schizophrenia and migration: a meta-analysis and review. Am J Psychiatry 2005; 162: 12-24.

4 Mulder CL, Koopmans GT, Selten JP. Emergency psychiatry, compulsory admissions and clinical presentation among immigrants to The Netherlands. Br J Psychiatry 2006; 188: 386-91.

5 Burnam MA, Hough RL, Karno M, Escobar Jl, Telles CA. Acculturation and lifetime prevalence of psychiatric disorders among Mexican Americans in Los Angeles. J Health Soc Behav 1987; 28: 89-102.

6 Vega WA, Kolody B, Aguilar-Gaxiola S, Alderete E, Catalano R, Caraveo-Anduaga J. Lifetime prevalence of DSM-III-R psychiatric disorders among urban and rural Mexican-Americans in California. Arch Gen Psychiatry 1998; 55: 771-8.

7 Takeuchi DT, Chung RC, Lin KM, Shen H, Kurasaki K, Chun CA, Sue S. Lifetime and twelve-month prevalence rates of major depressive disorders and dysthymia among Chinese Americans in Los Angeles. Am J Psychiatry 1998; 115: $1407-14$

8 Badawi M, Kramer M, Eaton W. Use of mental health services by households in the United States. Psychiatr Serv 1996; 47: 376-80.

9 Pérez-Rodriguez MM, Baca-García E, Quintero-Gutierrez J, Gonzalez G, Saiz-Gonzalez D, Botillo C, Basurte-Villamor I, Sevilla J, Gonzalez de Rivera JL. Demand for psychiatric emergency services and immigration. Findings in a Spanish hospital during the year 2003. Eur J Public Health 2006; 16: 383-7.

10 Coid JW, Kahtan N, Gault S, Jarman B. Ethnic differences in admissions to secure forensic psychiatry services. Br J Psychiatry 2000; 177: 241-7.

11 Mikton C, Grounds A. Cross-cultural clinical judgment bias in personality disorder diagnosis by forensic psychiatrists in the UK: a case-vignette study. J Personal Disord 2007; 21: 400-17.

12 Tyrer $\mathrm{P}$, Merson $\mathrm{S}$, Onyett $\mathrm{S}$, Johnson $\mathrm{T}$. The effects of personality disorder on clinical outcome, social networks and adjustment: a controlled clinical trial of psychiatric emergencies. Psychol Med 1994; 24: 731-40.

13 Baleydier B, Damsa C, Schutzbach C, Stauffer O, Glauser D. Comparison between Swiss and foreign patients' characteristics at the psychiatric emergencies department and the predictive factors of their management strategies. Encephale 2003; 29: 205-12.
14 Paris J. Cultural factors in the emergence of borderline pathology. Psychiatry 1996; 59: 185-92.

15 Martin-Santos R, Fonseca F, Domingo-Salvany A, Ginés JM, Ímaz ML, Navinés R, Pascual JC, Torrens M. Dual diagnosis in the psychiatric emergency room in Spain. Eur J Psychiatr 2006; 20: 147-56.

16 Pascual JC, Córcoles D, Castaño J, Ginés JM, Gurrea A, Martín-Santos R, Garcia-Ribera C, Pérez V, Bulbena A. Hospitalization and pharmacotherapy for borderline personality disorder in a psychiatric emergency service 2007. Psychiatr Serv 2007; 58: 1199-204.

17 American Psychiatric Association. Diagnostic and Statistical Manual of Mental Disorders (4th edn) (DSM-IV). APA, 1994.

18 Lyons J, Stutesman J, Neme J, Vessey JT, O'Mahoney MT, Camper HJ. Predicting psychiatric emergency admissions and hospital outcome. Med Care 1997; 35: 792-800.

19 Bulbena A, Pompei S, Ollé L, Coletas J. Medida de la gravedad de la enfermedad psiquiátrica [Measure of severity of psychiatric illness]. Arch Neurobiol 1997; 7: 69-79.

20 Morgan C, Mallet R, Hutchinson G, Leff J. Negative pathways to psychiatric care and ethnicity: the bridge between social science and psychiatry. Soc Sci Med 2004; 58: 739-52.

21 Swinnen SGHA, Selten JP. Mood disorders and migration. Meta-analysis. Br J Psychiatry 2007; 190: 6-10.

22 Shaw CM, Creed F, Tomenson B, Riste L, Cruickshank JK. Prevalence of anxiety and depressive illness and help seeking behaviour in African Caribbeans and white Europeans: two phase general population survey. BMJ 1999; 318: 302-5.

23 Murphy HBM. Comparative Psychiatry. Springer, 1982.

24 Linehan MM. Cognitive-Behavioural Treatment of Borderline Personality Disorder. Guilford Press, 1993.

25 Millon T. Sociocultural conceptions of the borderline personality. Psychiatr Clin North Am 2000; 23: 123-36.

26 Paris J. Social Factors in the Personality Disorders: A Biopsychosocial Approach to Etiology and Treatment. Cambridge University Press, 1996.

27 Van de Vijver F, Leung K. Methods and Data Analysis for Cross-cultural Research. Sage, 1997.

28 Harkness JA, Van de Vijver FJR, Mohler PP. Cross-cultural Survey Methods. Wiley, 2003

29 Chavira DA, Grilo CM, Shea MT, Yen S, Gunderson JG, Morey LC, Skodol AE, Stout RL, Zanarini MC, MCGlashan TH. Ethnicity and four personality disorders. Compr Psychiatry 2003; 6: 483-91.

30 Mellsop G, Varghese F, Joshua S, Hicks A. The reliability of Axis II of DSM-III. Am J Psychiatry, 1982; 139: 1360-1.

31 Alarcon RD, Foulks EF, Vakkur M. Personality Disorders and Culture: Clinical and Conceptual Interactions. Wiley, 1998.

32 Warner MD, Peabody CE. Reliability of diagnoses made by psychiatric residents in a general emergency department. Psychiatr Serv 1995; 46: 1284-6. 